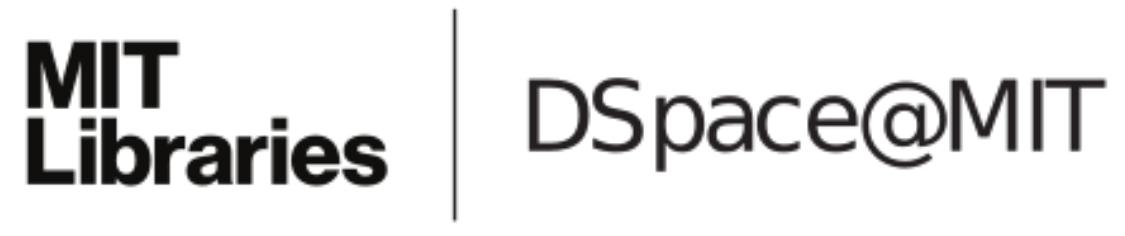

MIT Open Access Articles

Lump detection with a gelsight sensor

The MIT Faculty has made this article openly available. Please share how this access benefits you. Your story matters.

Citation: Xiaodan Jia, Rui Li, M. A. Srinivasan, and E. H. Adelson. "Lump Detection with a Gelsight Sensor." 2013 World Haptics Conference (WHC) (n.d.).

As Published: http://dx.doi.org/10.1109/WHC.2013.6548404

Publisher: Institute of Electrical and Electronics Engineers (IEEE)

Persistent URL: http://hdl.handle.net/1721.1/86139

Version: Author's final manuscript: final author's manuscript post peer review, without publisher's formatting or copy editing

Terms of use: Creative Commons Attribution-Noncommercial-Share Alike 


\section{Lump Detection with a GelSight Sensor}

\author{
Xiaodan (Stella) Jia* \\ Perceptual Science Group \\ MIT
}

\author{
Rui $\mathrm{Li}^{\dagger}$ \\ Perceptual Science Group \\ MIT
}

\author{
Mandayam A. Srinivasan \\ The Touch Lab \\ MIT
}

Edward H. Adelson
Perceptual Science Group
MIT

\begin{abstract}
A GelSight sensor is a tactile sensing device comprising a clear elastomeric pad covered with a reflective membrane, coupled with optics to measure the membrane's deformations. When the pad is pressed against an object's surface, the membrane changes shape in accord with mechanical and geometrical properties of the object. Since soft tissue is more compliant than hard tissue, one can detect an embedded lump by pressing the GelSight pad against the tissue surface and observing the hump that forms over the lump. We tested this system's sensitivity by constructing phantoms of soft rubber with hard embedded lumps. The system is quite sensitive; for example it could detect a $2 \mathrm{~mm}$ lump at a depth of $5 \mathrm{~mm}$. The sensor was more sensitive than previous tactile lump detectors. It was also better than human observers using their fingertips. Such a capability could help in tumor screening, and could augment the sensory information available in telemedicine or minimally invasive surgery.
\end{abstract}

Index Terms: H.1.2 [Models and Principles]: User/Machine System-Human Information Processing; H.5.2 [Information Interfaces and Presentation]: User Interface-Haptics I/O;

\section{INTRODUCTION}

\subsection{Background}

The sense of touch is important in medical diagnosis and treatment. In particular, the ability to detect variations in tissue softness through palpation is an essential component in the routine examination for breast, testicular, or prostate cancer [4]. In addition, the ability to discriminate hard and soft tissues is an important part of open surgery and would be helpful if it can augment the sensory data available in minimally invasive surgery. In breast lumps examination, for example, in which the hard tissue (lump) embedded in the soft tissues [3], tactile sensation plays a significant role in the first stage of examination. From the clinical data in [7], [11], [12], we can know that lumps are significantly stiffer than surrounding tissues. Such contrast of stiffness can assist doctors in the localization and assessment of lumps during lump examinations, when the doctors' fingertips are in direct contact with the tissue [6].

Detection of lumps using tactile sensors is still a challenging problem. Gwilliam et al. [6], studied lump detection using an artificial touch sensor based on a capacitive array [13]. They constructed phantoms of soft silicone rubber containing hard embedded balls of various sizes and depths, and derived pressure distributions as they pressed the sensor on the phantom. They were able to achieve sensitivity somewhat greater than that of human subjects tested with the same phantoms. We undertook a similar study using a novel sensor, which measures the distortion of a soft elastomeric pad as it is pressed against a surface.

\footnotetext{
*e-mail: xiaodan@mit.edu

†e-mail: rui@mit.edu

†e-mail:srini@mit.edu

§e-mail:adelson@csail.mit.edu
}

\subsection{The GelSight Sensor}

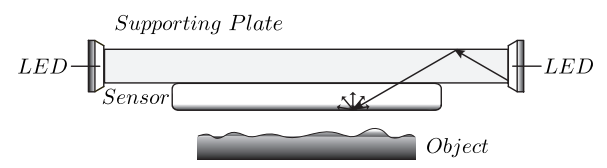

Figure 1: Basic principle of the Gelsight sensor. There are four main components for the GelSight sensor: an elastomer with the opaque reflective membrane on top, supporting plate which provides the support for the soft elastomer while pushing against the object, optics and LEDs which provide illumination for the sensor, and the camera in the back to capture the deformation images under the illumination from different directions. Sometimes, the optics can functions as a supporting plate as well.

Johnson and Adelson [9] and Johnson et al. [10] described a tactile sensor, called Gelsight, which is based on a clear elastomeric pad covered by a reflective membrane. A camera is placed behind the pad and views the membrane as it is illuminated by LEDs from various directions (see Figure 1). When the pad is pressed against a rigid surface, the membrane takes on the surface's 3D topography, allowing the camera to record a shaded image indicative of that topography (see Figure 2). Through the use of photometric stereo [14], [1], the sensor can produce a detailed image of the membrane's 3D shape. As shown in Figure $2 \mathrm{a}$ and $2 \mathrm{~b}$, an Oreo cookie is pressed against the GelSight sensor. Depending on the task, this image can be used directly, or can be used to infer pressure distributions or other aspects of the object being probed. Johnson et al. [10] discussed the accuracy of different reconstruction approaches and we employ the most accurate one. In the case of lump detection, the problem is to detect the presence of a stiffer tissue within a volume of normal tissue. As will be described, this can be detected by measuring the change in surface geometry under increasing force.

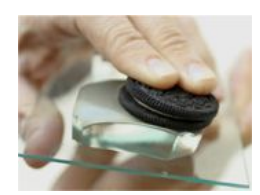

(a)

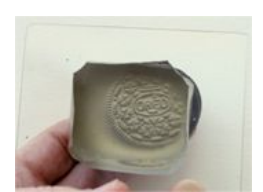

(b)

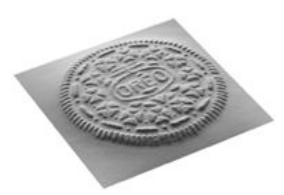

(c)
Figure 2: (a) A cookie is pressed against the membrane of a GelSight sensor. (b) The membrane is distorted, as shown in this view from beneath. (c) The cookie's 3D shape is reconstructed using photometric stereo and rendered at a different viewpoint. [9] 


\section{LUMP Detection}

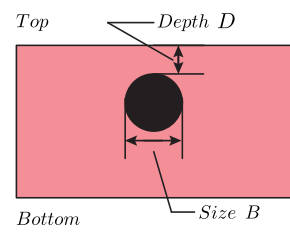

Figure 3: Physical illustration of a phantom with a spherical lump (black) of diameter B embedded at depth D from the top.

Following Gwilliam et al. [6], we constructed phantoms using Ecoflex 0030 silicone rubber from Smooth-On Inc. This rubber has a Shore 00 harness of 30, and is similar to soft human tissue. Within the phantoms we placed Delrin spheres, which we take as lumps, of various diameters, embedded at various depths. The dimensions of the phantoms are shown in Figure 3, and the range of lump sizes and depths are shown in Table 1. Sizes were 2, 3, 5, 8, or $9.5 \mathrm{~mm}$, while depths were $1,2,3,4,5$, or $6 \mathrm{~mm}$. A phantom with, for example, a $3 \mathrm{~mm}$ lump at $5 \mathrm{~mm}$ depth is denoted as B3-D5. We also made blank phantoms containing no lumps.

Table 1: All phatoms with lumps

\begin{tabular}{ccccccc}
\hline$[\mathrm{mm}]$ & Ball & 2 & 3 & 5 & 8 & 9.5 \\
Depth & & B2 & B3 & B5 & B8 & B9.5 \\
\hline & & & & & & \\
1 & D1 & D1-B2 & D1-B3 & D1-B5 & D1-B8 & D1-B9.5 \\
2 & D2 & D2-B2 & D2-B3 & D2-B5 & D2-B8 & D2-B9.5 \\
3 & D3 & D3-B2 & D3-B3 & D3-B5 & D3-B8 & D3-B9.5 \\
4 & D4 & D4-B2 & D4-B3 & D4-B5 & D4-B8 & D4-B9.5 \\
5 & D5 & D5-B2 & D5-B3 & D5-B5 & D5-B8 & D5-B9.5 \\
6 & D6 & D6-B2 & D6-B3 & D6-B5 & D6-B8 & D6-B9.5
\end{tabular}

\subsection{GelSight Experiment}

\subsubsection{Experiment Setup}

The GelSight sensor system was housed in a box and mounted on a vertical shaft, as shown in Figure $4 \mathrm{a}$. The phantom rested on a digital scale, and the sensor was pressed against the phantom with the desired force.

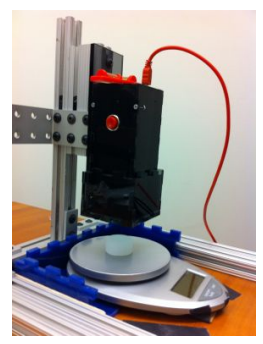

(a)

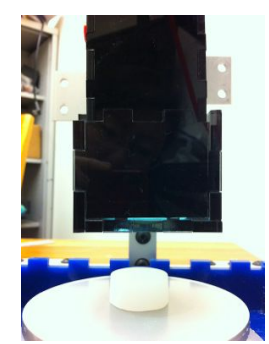

(b)
Figure 4: Setup of the GelSight experiment for lump detection. (a) Side view of the overall system. (b) Closer front view of the GelSight device and the phantom.

Figure 5 shows how the sensor is able to detect the presence of a lump. On the left is a phantom in its resting state, with the embedded lump shown as a black disc. The GelSight elastomeric pad is placed above the phantom, and then pressed against it in a downward direction. Both the phantom and the pad distort as the pressure is applied: they bulge outward, and the interface between them (including the reflective membrane) is deformed according to the distribution of stiffness. Since a hard lump is less compliant than soft tissue, it produces a hump shaped distortion in the membrane. The geometry of these changes is measured by photometric stereo and converted to a depth image. For the detailed reconstruction algorithm, please refer to [8].

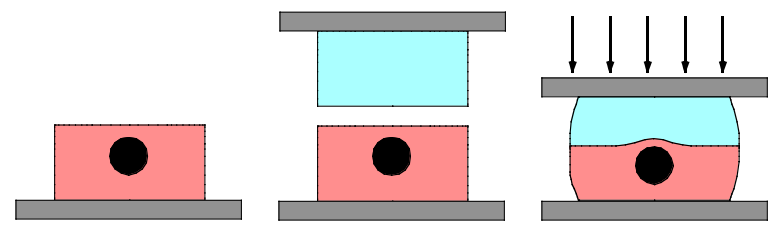

Figure 5: Illustration of the GelSight sensor's deformation when the sensor (blue) is applied against a phantom (purple) with a lump embedded.

Our device, shown in Figure 4, is derived from the handheld sensor described by Johnson et al. [9]. It consists of a rectangular housing $(62 \mathrm{~mm} \times 62 \mathrm{~mm} \times 150 \mathrm{~mm})$ containing a Flea2 camera (Point Grey Systems), as well as the lighting, the control electronics, and a circular glass plate supporting the elastomeric pad. The plate was illuminated by 6 LEDs which are lit sequentially to produce the images needed for photometric stereo. The pads were made from a thermoplastic gel rubber (a styrenic block copolymer, SST-RN, supplied by Crinnis, Inc.). They were $35 \mathrm{~mm}$ in diameter, $4 \mathrm{~mm}$ thick, and had softness similar to that of the phantoms. The pads were covered with a thin membrane of the same gel rubber mixed with fine aluminum powder, producing a coating that was opaque, gray, and matte in appearance.

\subsubsection{Approach of Using GelSight in Lump Detection}

To test performance of the GelSight sensor for lump detection, we experiment with phantoms that have lumps of different sizes embedded at different depths in the tissue and with different forces applied on each phantom. For each trial, a particular phantom was placed below the GelSight device, and the pad was pressed against the phantom with a given amount of force. Following, Gwilliam et al. [6], we express the force in gram equivalent units. Table 2 shows the conversion to Newton. We also show the pressure for each condition, estimated by dividing by the contact area of 962 $\mathrm{mm}^{2}$.

Table 2: Force and pressure applied in lump detection experiments

\begin{tabular}{cccccc}
\hline Force in grams [g] & 500 & 1000 & 1500 & 2000 & 2500 \\
Force in Newtons [N] & 4.90 & 9.81 & 14.71 & 19.61 & 24.52 \\
$\begin{array}{c}\left.\text { Pressure[10-3 N/mm } \mathrm{mm}^{2}\right] \\
\text { over a area of } 962 \mathrm{~mm}^{2}\end{array}$ & 5.09 & 10.19 & 15.29 & 20.39 & 25.49 \\
\hline
\end{tabular}

The depth image on a given trial can be considered to have two components: the baseline image that results from pressing against a blank phantom, and the distortion from baseline that is caused by the lump. For each force, we obtained a baseline image by averaging the depth images of 10 blank phantoms. Then, for further experiments we subtracted the baseline from the observed depth image, giving us a residual image containing the distortion caused by the lump.

Figure $6 \mathrm{~b}$ shows the residual images obtained with a force of $2500 \mathrm{~g}$, using all 30 of the phantoms, with all possible combinations 
of sizes and depths. (Note that the lumps are not perfectly centered beneath the sensor, because of random variation in the relative positioning from trial to trial. We retained this positional variation in the depth images since it corresponds to a realistic condition in which lump location is not exactly known). Figure 6a shows a scale bar mapping the color into depth, and Figure $6 \mathrm{c}$ shows a set of 10 measurements taken with blank phantoms.

The residual images in Figure 6b clearly reveal the presence of the lump for most of the conditions. Large lumps and shallow depths (upper right corner) lead to prominent bulges. As the lumps become smaller and the depth becomes greater, the bulge becomes more subtle (lower left corner). To convert this visual impression to a quantitative measurement, we devised a classifier that could distinguish between blank and non-blank phantoms.

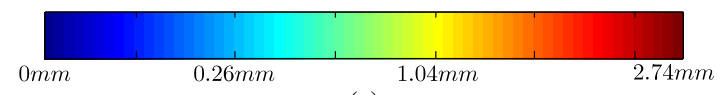

(a)

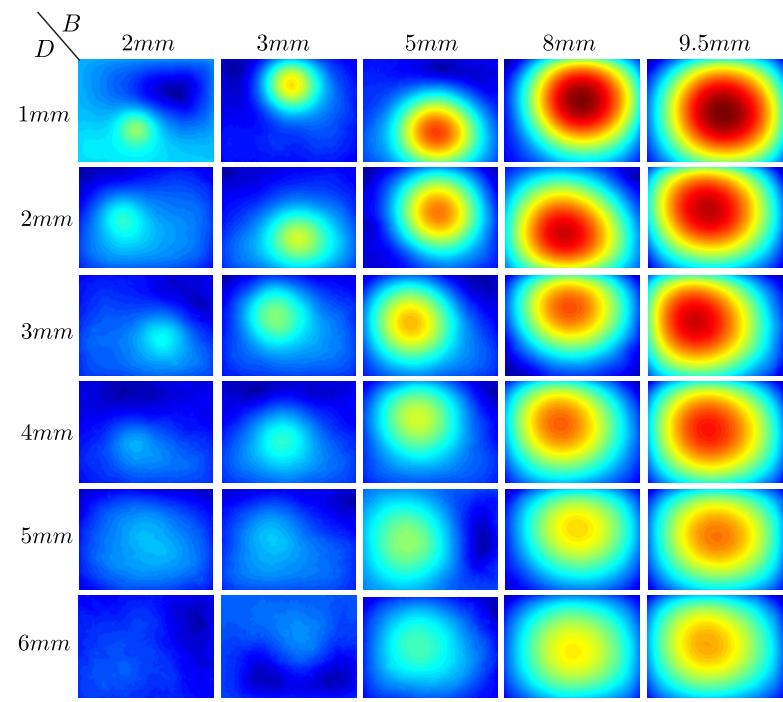

(b)
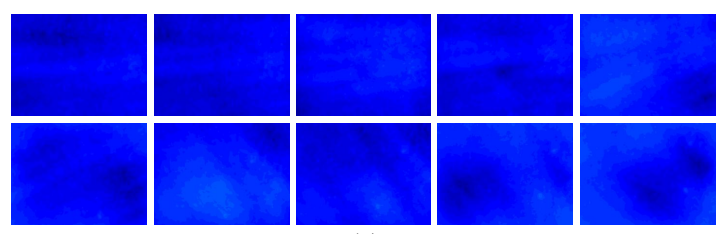

(c)

Figure 6: (a) Colorbar mapping color to the depth in millimeter. (b) Depth images of all phantoms with $2500 \mathrm{~g}$ force. Each row represents phantoms with lumps at different depths. From up to down, the depths are D1, D2, D3, D4, D5, and D6, respectively. Each column represents phantoms with lumps of different sizes. From left to right, the size is B2, B3, B5, B8 and B9.5, respectively. (c) Depth images of blank phantoms with $2500 \mathrm{~g}$ force.

We found that a variety of features could be used successfully in the classification. The particular algorithm we settled on was as follows. We computed the residual image by subtracting the observed depth image from the average image of 10 blank phantoms at the same force. We retained all non-negative values, since the presence of a lump is signaled by larger values over the lump. Then we computed the mean and variance of the values taken across the residual image. By using a standard support vector machine classifier [2] with supervised learning on the images with and without lumps, we determined the linear decision boundary for that force condition. For $2500 \mathrm{~g}$ force, the boundary was

$$
1.21 m_{R}+0.63 v_{R}=6 .
$$

where $m_{R}$ and $v_{R}$ are the mean and variance of the residual image. For any pair of $\left(m_{R}, v_{R}\right)$ above the line, the classification result is that there is a lump present, and vice versa. Performance of the classifier is shown and discussed in more detail in section 3 .

\subsection{Human Psychological Experiment}

The purpose of this experiment is to see how humans perform in the lump detection tasks. We tested the performance of human subjects using the same phantoms as in the GelSight experiment. We have 4 naive human subjects, each of which was given a few trials of practice and then was asked to distinguish blank phantoms from those with lumps. We used the method called two alternative forced choice: for each trial, the subject was presented with two phantoms, had 12 seconds to touch the phantoms using a single finger and choose the one containing the lump. To restrict the amount of applied force, the phantoms were mounted on a counterbalanced arm, which prevented the exertion of forces greater than $2000 \mathrm{~g}$ force. The size of human fingertips is about $1 / 10 \sim 1 / 6$ of the contacting area between the phantom and the GelSight sensor, or $96 \mathrm{~mm}^{2} \sim 160 \mathrm{~mm}^{2}$. Each subject ran 10 trials for each phantom pair, so there are 40 trials in total for all 4 human subjects.

\section{Results ANd Discussions}

\subsection{Results for GelSight and for Human Psychophysics}

Table 3 shows the results of lump detection for the GelSight sensor with the force of $2500 \mathrm{~g}$. The yellow blocks indicate the conditions under which the system could correctly distinguish between phantoms with and those without lumps. The system can tell whether there is a lump in all conditions except for two involving the greatest depth and smallest lumps: D6-B2 and D6-B3.

Table 3: Performance of the GelSight sensor in lump detection for different depths and lump sizes. The yellow area denotes correct detection.

\begin{tabular}{ccccccc}
\hline$[\mathrm{mm}]$ & Ball & 2 & 3 & 5 & 8 & 9.5 \\
Depth & & B2 & B3 & B5 & B8 & B9.5 \\
\hline & & & & & & \\
1 & D1 & D1-B2 & D1-B3 & D1-B5 & D1-B8 & D1-B9.5 \\
2 & D2 & D2-B2 & D2-B3 & D2-B5 & D2-B8 & D2-B9.5 \\
3 & D3 & D3-B2 & D3-B3 & D3-B5 & D3-B8 & D3-B9.5 \\
4 & D4 & D4-B2 & D4-B3 & D4-B5 & D4-B8 & D4-B9.5 \\
5 & D5 & D5-B2 & D5-B3 & D5-B5 & D5-B8 & D5-B9.5 \\
6 & D6 & D6-B2 & D6-B3 & D6-B5 & D6-B8 & D6-B9.5 \\
\hline
\end{tabular}

Table 4: Performance of humans in lump detection. The yellow area denotes correct detection.

\begin{tabular}{ccccccc}
\hline$[\mathrm{mm}]$ & Ball & 2 & 3 & 5 & 8 & 9.5 \\
Depth & & B2 & B3 & B5 & B8 & B9.5 \\
\hline & & & & & & \\
1 & D1 & D1-B2 & D1-B3 & D1-B5 & D1-B8 & D1-B9.5 \\
2 & D2 & D2-B2 & D2-B3 & D2-B5 & D2-B8 & D2-B9.5 \\
3 & D3 & D3-B2 & D3-B3 & D3-B5 & D3-B8 & D3-B9.5 \\
4 & D4 & D4-B2 & D4-B3 & D4-B5 & D4-B8 & D4-B9.5 \\
5 & D5 & D5-B2 & D5-B3 & D5-B5 & D5-B8 & D5-B9.5 \\
6 & D6 & D6-B2 & D6-B3 & D6-B5 & D6-B8 & D6-B9.5 \\
\hline
\end{tabular}


Table 4 shows the performance of the human observers by averaging the performance of all trials for each phantom. If each phantom was detected correctly for at least $75 \%$ of the times (or 30 out of 40 times in our experiment), we denote that the lumps can be detected. As expected, there is a tradeoff between the depth and size of the lump: for the same depth, the larger the lump size, the easier it is to detect the lump, and for the same lump size, the smaller the depth, the easier it is to detect the lump. A total of 11 conditions are below threshold for human detection: for example, a lump of $2 \mathrm{~mm}$ could not be detected at a depth of $2 \mathrm{~mm}$ or deeper for humans, and a lump of $8 \mathrm{~mm}$ could not be detected at a depth of $6 \mathrm{~mm}$ or deeper.

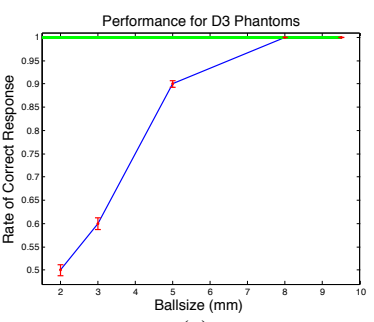

(a)

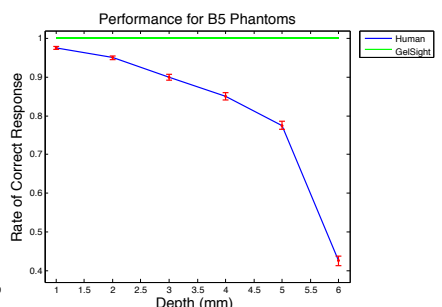

(b)
Figure 7: Performance comparisons of the GelSight sensor and humans in lump detection for (a) D3 phantoms, and (b) B5 phantoms.

Figure 7 shows the performance comparison of the GelSight sensor and humans. It can be seen that the GelSight sensor is much more sensitive than the human subjects with the same lump size or depth. For the same lump size, e.g., $3 \mathrm{~mm}$, the GelSight sensor can detect it even at a large depth of $5 \mathrm{~mm}$ as compared to $2 \mathrm{~mm}$ for humans. For the same depth, e.g., $5 \mathrm{~mm}$, the GelSight sensor can detect balls with size as small as $2 \mathrm{~mm}$ as compared to $5 \mathrm{~mm}$ for humans. All above comparisons are done with the conditions that the GelSight sensor applies a much smaller pressure than humans do on the phantoms (the pressue for GelSight is about 1/8 1/5 of that for humans), which makes the GelSight sensor even better in performance compared to humans.

\subsection{Performance comparison of the GelSight Sensor and the DigiTacts Sensor}

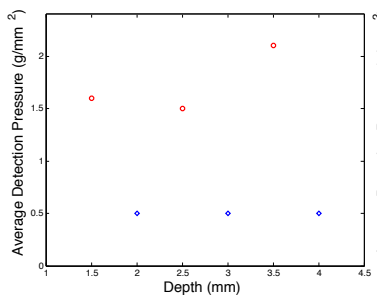

(a)

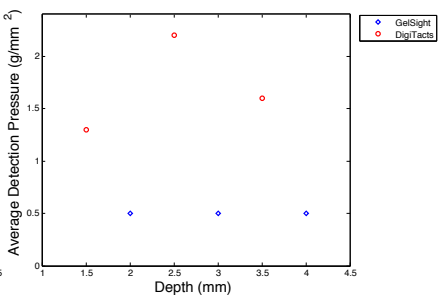

(b)
Figure 8: Detection pressure as a function of lump depth for the GelSight sensor and the DigiTacts sensor. (a) B5 phantoms for GelSight and B6.5 phantoms for DigiTacts. (b) B9.5 phantoms for both GelSight and DigiTacts

Gwilliam et al. [6] introduced a lump detection experiment using the DigiTacts II capacitive sensor. Phantoms used in their experiments are similar to what we have used for testing the GelSight sensor, but they focused their work on larger lumps with shallower depths than we did. The smallest lumps they tested were $6.5 \mathrm{~mm}$

and the deepest depth tested was $3.5 \mathrm{~mm}$. They did not use fixed forces, as we did, but we can compare their sensitivity to ours by considering the pressure required for phantoms of similar parameters. For example, we can compare their phantom with a $6.5 \mathrm{~mm}$ lump size at $3.5 \mathrm{~mm}$ depth to our phantom of $5 \mathrm{~mm}$ lump size at $4 \mathrm{~mm}$ depth. In these conditions, their capacitive sensor required a pressure of about $2.2 \mathrm{~g} / \mathrm{mm}^{2}$ for detection, whereas with ours the detection pressure was below $0.5 \mathrm{~g} / \mathrm{mm}^{2}$. Thus the pressure required with GelSight was less than $1 / 4$ of that required with the capacitive sensor. In addition, the fact that we were able to detect lumps that were much smaller and much deeper indicates the improved sensitivity of GelSight in this task.

\subsection{Discussions on the Resulted Phantom Images}
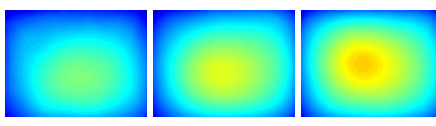

(a)
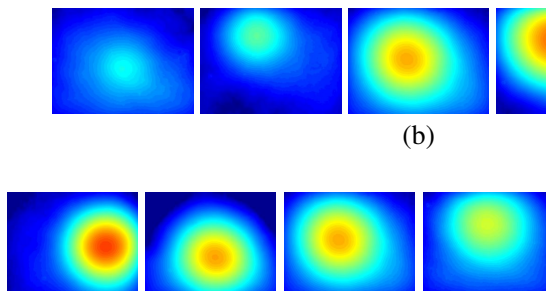

(c)

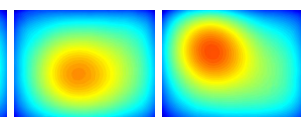

Figure 9: (a) Depth image of D3-B5 pressed with different forces for the blank phantom. From left to right, the forces are $500 \mathrm{~g}$, $1000 \mathrm{~g}, 1500 \mathrm{~g}, 2000 \mathrm{~g}$, and 2500g, respectively. (b) Depth image of D3 phantoms with lumps of different sizes pressed with $2500 \mathrm{~g}$ force. From left to right, the sizes of the lumps are B2, B3, B5, B8, and B9.5 respectively. (c) Depth image of B5 phantoms with lumps embedded at different depths pressed at $2500 \mathrm{~g}$ force. From left to right, the depths are D1, D2, D3, D4, D5, and D6 respectively.

\subsubsection{Phantoms with different applied forces}

To test whether an applied force can influence the detection results, we select phantom with medium lump size (B5) embedded at medium depth (D3) as an illustration. Figure 9a shows the depth images of D3-B5 applied by different forces, 500g, 1000g, 1500g, $2000 \mathrm{~g}, 2500 \mathrm{~g}$, respectively. From all the depth images shown in Figure $9 \mathrm{a}$, we can see that the larger the force, the stronger the contrast in the depth image.

\subsubsection{Phantoms with lumps of different sizes}

To see how sizes of lumps influence the depth image, we pick phantoms with lumps embedded at a medium depth (D3 phantoms) while pressed with the same force $(2500 \mathrm{~g}$ force) as illustration. From Figure 9b, it can infer that the larger the lumps are, the easier to detect whether the lump is present or not.

\subsubsection{Phantoms with lumps embedded at different depths}

To see how the depths of lumps can influence the depth image, we pick phantoms with lumps of medium size (B5 phantoms). All the phantoms are pressed with the same force (2500g force). The depth images are shown in Figure 9c. Simply by looking at the depth images, the contrast of the lump from surroundings is getting less as the depth increases. 


\section{Conclusion}

In this paper we have described how a GelSight sensor, built with a soft elastomeric pad, can be used for lump detection. When pressed against a soft rubber phantom containing a hard embedded lump, the membrane of the sensor distorts, exhibiting a hump that indicates the presence of the lump beneath the surface. We were able to discriminate the lump phantoms from blank phantoms even when the lumps were $2 \mathrm{~mm}$ in diameter and $5 \mathrm{~mm}$ deep. This sensitivity is substantially greater than that which was previously demonstrated by Gwilliam et al. [6] with a capacitive sensor. In addition, it is substantially greater than that achieved by human subjects with the same phantoms. Our experiments were done under highly simplified conditions, and our devices form factor is inappropriate for deployment in a real world setting. Nonetheless, the fact that we are able to achieve such high sensitivities indicates that the GelSight technology has promise for applications involving the detection of lumps in soft tissues.

\section{ACKNOWLEDGEMENTS}

This work was supported in part by National Science Foundation grant 6922551. The authors thank Kimo Johnson for providing the portable device of GelSight sensor, Wenzhen Yuan for the asssitance in phantoms manufacturing.

\section{References}

[1] S. Barsky and M. Petrou. The 4-source photometric stereo technique for three-dimensional surfaces in the presence of highlights and shadows. Pattern Analysis and Machine Intelligence, IEEE Transactions on, 25(10): 1239 - 1252, oct. 2003.

[2] N. Cristianini and J. Shawe-Taylor. An Introduction to Support Vector Machines and Other Kernel-based Learning Methods. Cambridge University Press, Cambridge, first edition edition, 2000.

[3] P. Dario. Tactile sensing: Technology and applications. Sensors and Actuators A: Physical, 26(13):251 - 256, 1991. ¡ce:title ¿Proceedings of Eurosensors IV held in jointly with Sensoren Technologies und Anwendung ${ }_{i} /$ ce:title $i$.

[4] L. E. Eberman and M. E. Finn. Enhancing clinical evaluation skills: Palpation as the principal skill. Athletic Training Education Journal, 5:170-175, 2010.

[5] N. J. Ferrier and R. W. Brockett. Reconstructing the shape of a deformable membrane from image data, 2000.

[6] J. Gwilliam, Z. Pezzementi, E. Jantho, A. Okamura, and S. Hsiao. Human vs. robotic tactile sensing: Detecting lumps in soft tissue. In Haptics Symposium, 2010 IEEE, pages 21 -28, march 2010.

[7] K. Hoyt, B. Castaneda, M. Zhang, P. Nigwekar, P. A. DiSantagnese, J. V. Joseph, J. Strang, D. J. Rubens, and K. J. Parker. Tissue elasticity properties as biomarkers for prostate cancer. Cancer Biomarkers, 4(45):213-225, 2008.

[8] X. Jia. Lump detection using elastomeric sensors. Master's thesis, Massachusetts Institute of Technology, 2012.

[9] M. K. Johnson and E. H. Adelson. Retrographic sensing for the measurement of surface texture and shape. In Computer Vision and Pattern Recognition (CVPR), pages 1070-1077, 2009.

[10] M. K. Johnson, F. Cole, A. Raj, and E. H. Adelson. Microgeometry capture using an elastomeric sensor. ACM Transactions on Graphics (Proc. ACM SIGGRAPH), 30(4):461-468, 2011.

[11] T. Krouskop, T. Wheeler, F. Kallel, and B. Garra. Elastic moduli of breast and prostate tissues under compression. Ultrasonic imaging, 20(4):260-274, Dec. 1998.

[12] S. Phipps, T. Yang, F. Habib, R. Reuben, and S. McNeill. Measurement of tissue mechanical characteristics to distinguish between benign and malignant prostatic disease. Urology, 66(2):447 - 450, 2005.

[13] P. P. Systems. Digitacts ii, tactile array sensor evaluation kit with digital output.

[14] R. J. Woodham. Gradient and curvature from photometric stereo including local confidence estimation. Journal of the Optical Society of America A, 11:3050-3068, 1994. 